\title{
LINEAR MODELLING AND FOURIER SPECTROSCOPY OF STRESS- RELAXATION IN THE CERVICAL SPINE
}

\author{
Andrew D Holmes, Ph.D. and Jing Fang*, Ph.D. \\ Department of Orthopaedic Surgery, University of Hong Kong, Pokfulam Road, Hong Kong. \\ *Department of Mechanics, Peking University, Beijing 100871, People's Republic of China. \\ E-mail: aholmes@hkucc.hku.hk
}

\begin{abstract}
Single joints of the cervical spine were subject to axial compressive loads and the load relaxation recorded for 15 minutes. Isochronal plots of stress against strain were plotted for each specimen and showed high linear correlation coefficients $(>0.955)$. Accordingly, a linear model was chosen and fitted to data obtained from a fuller range ( 3 hours) of the complete relaxation curve. The relaxation times extracted from this model were approximately 10 minutes and 5 hours.

Fourier transform of the relaxation curve allows the storage modulus and the loss modulus of the relaxation spectrum to be shown separately as functions of frequency. Fourier analysis shows that the cervical spine has a wide and smooth distribution of relaxation times. Examination of the higher frequency components of the relaxation in the region of 1 to $20 \mathrm{~Hz}$ show the loss modulus to be almost flat and tending towards zero.

This work shows that while the disc is capable of dissipating a large proportion of the applied strain energy, it can only do this slowly, and the discs of the cervical spine are inefficient as 'shock absorbers' of axial loads.
\end{abstract}

Introduction: The intervertebral discs show marked viscoelastic behavior, and the more rapidly they are loaded then the greater the resistance they will show to the applied load (Smeathers, 1989). This has significant implications in understanding how the spinal joints work both individually and within the system of the spinal column. Work on the lumbar spine (Smeathers and Joanes, 1988) has shown that the $5 \mathrm{~Hz}$ resonance observed by other experimenters in vivo (e.g., Panjabi et al., 1986) is not reproduced in experiments performed in vitro under axial compression, and that the shortest time scales over which the lumbar spine shows any appreciable dissipation of strain energy is somewhere in the region of tens of minutes (Holmes, 1991).

The cervical spine is another part of the spine where the time dependent mechanical properties of the structure is likely to be an important feature, whether the source of loading be from external oscillators (vibration of vehicles, pneumatic tools, etc.) or from impact loading on the cranium. Compression is likely to be a main loading mode, and the suggested second stiffest buckling axis in the cervical spine (King-Liu and Guo Dai, 1989) also would indicate that the compressive forces in the cervical spine may still be greatest even if the loading is not a true axial compression.

Methods: 10 spinal segments were used from young male subjects( 25 to 39 years old). Two adjacent vertebrae and the intervertebral disc connecting them were attached to a pair of loading plates using short bone screws, and mounted in a material testing machine. Care was taken at all times during the preparation and mounting of the specimen to keep it thouroughly moistened with physiological saline.

The experiments were separated into two parts: firstly to examine several relaxation curves from different strain values for the same segment to plot isochronal curves, and secondly to examine the long term relaxation of cervical motion segments. The relaxation curves for the isochronal plots were recorded over 15 minutes on a $x-y$ plotter, and the 5 specimens used for this part of the experiments allowed to recover (in an unstressed state) for 45 minutes between each loading session. The data for long term relaxation was digitised through a 12 bit converter at $20 \mathrm{~Hz}$ and logged automatically on a microprocessor over three hours or longer. A preliminary test performed on an additional single motion segment loaded three times to the same initial stress showed that this resting time of 45 minutes allowed the segment to show reproducible relaxation curves indicating the segment had essentially 'forgotten' its past loading experience (Dorrington, 1980).

Results \& Discussion: A typical isochronal plot for a single specimen at times of 0,1 , and 10 minutes is shown in figure 1 . The lines marked on this figure are those obtained by linear regression analysis, and the lowest value obtained for the linear correlation coefficient was found to be 0.955 for all of the specimens tested (average $r=0.983$ ). Although the origin of the regression lines from these isochronal plots do not coincide with that of the $x-y$ axis, this is to be expected as the isochronal plot is simply a reflection of the quasi-static stressstrain properties of the segment. A material that exhibits non-linear stress-strain properties may still exhibit linear stress relaxation, providing that (a) the shape of the isochronal plot for $\mathrm{t}=0$ is similar to that seen in quasi-static loading and (b) that the shape of the isochronal plot remains constant with increasing time (Holmes, 1991). 


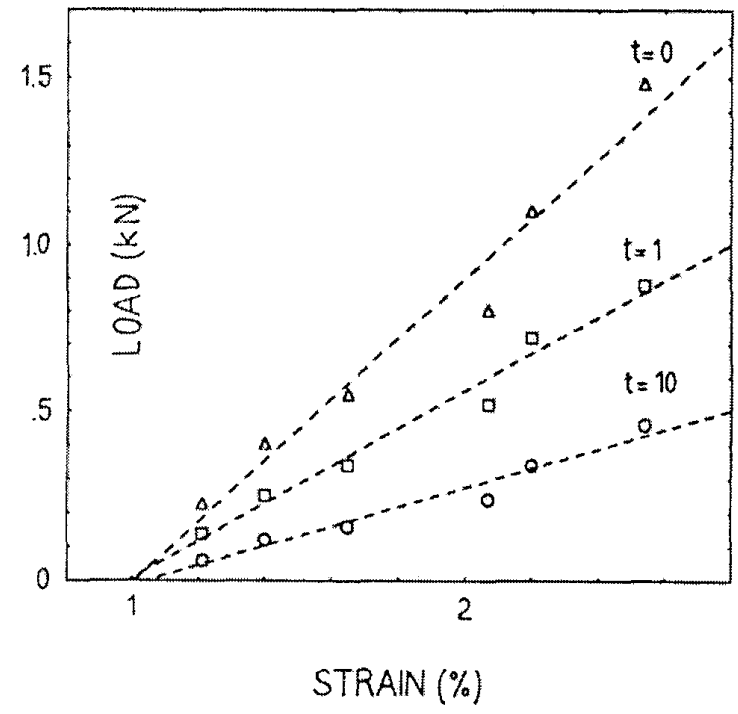

Figure 1

The results shown in the isochronal plots justify the modelling of the data by a linear system, using the criteria that the disc is poroelastic (will not return to it's full original height) and that a single relaxation time is insufficient given the range over which relaxation is observed. On the basis of this, the simplest viscoelastic model conforming to the observed experimental phenomena was used. This consisted of two Maxwell elements combined in series with a degenerate spring, and a plastic (Coulomb friction plate) element in parallel with one of the Maxwell elements. As the system is linear, then the form of the solution for this type of model can be found by superposition of standard solutions for viscoelastic models, as detailed in many standard texts (e.g., Shames and Cozzarelli, 1992). The analytical solution of the model is determined by two relaxation times and a finite asymptotic load, given by

$$
\sigma(t)=A e^{-\alpha t}+B e^{-\beta t}+C
$$

Relaxation curves given by this equation were fitted to the long term stress relaxation data collected, and the curve fitting procedure optimised by the downhill simplex method. This produced a reasonably accurate fit along the length of the curve: the lowest value of the root mean square (rms) deviation per data point between the experimental and theoretical curves was $5.6 \mathrm{~N}$ (average $3.2 \mathrm{~N}$ ). The two relaxation times extracted from the fitted theoretical curves were of the order of $10 \mathrm{~min}$ and $5 \mathrm{hr}$. Averaged values for all the specimens (with the standard deviation quoted in parenthesis) are $8.70 \mathrm{~min}(1.29 \mathrm{~min})$ and $4.31 \mathrm{hr}(1.01 \mathrm{hr})$. Linear analysis of stress relaxation curves is somewhat disadvantaged in that it typically presupposes the form of the data obtained by the modelling process, as above, and can only describe it in very simple terms. While it is useful for establishing 'ballpark' values for relaxation times, the relaxation will be more generally made up of a continuous spectrum of relaxation times.

Fourier transform mechanical spectroscopy (FTMS) described by Arridge and Barham (1986) transforms the data into separate spectrums of loss and storage moduli, continuously defined for a frequency range corresponding to endpoints of the data acquisition time and the duration of the experiment. This makes no assumption on the form of the data (linear or non-linear) and is an accurate and convenient method for examining the stress relaxation data. An empirical correction to counter aliasing effects that occur when a discrete Fourier transform (DFT) is performed was formulated by Aspden (1991), and a similar method including the aliasing correction to the loss modulus was used for the analysis of the long term relaxation specimens. This was performed on a microcomputer using a standard FFT algorithm. The Fourier transform procedure is extremely sensitive to noise in the signal, so the data was smoothed using cubic B-splines before passing to the DFT. An example of the resulting relaxation spectrum is shown in figure 2 .

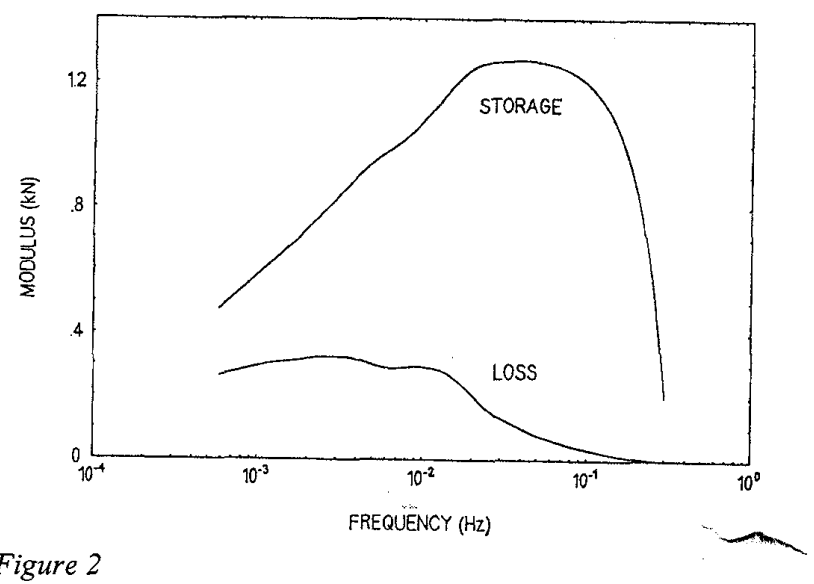

The sudden levelling off and then steep decrease in the storage modulus at higher frequencies (greater than $0.1 \mathrm{~Hz}$ ) is not a true effect, but simply an artefact due to aliasing. The same but opposite effect occurs in the loss modulus but this is compensated for by the empirical correction mentioned above (Aspden, 1991). It can be seen that neither the storage or loss moduli show any strong relaxation features at any certain frequency, and both spectra are basically smoothly defined throughout the frequency domain. Separate examination of the high frequency end of the relaxation spectrum for another specimen is showed that the $5 \mathrm{~Hz}$ resonance seen in the spine in vivo is not obvious in these axial compression tests, and that generally the value of the loss modulus is very low. At high frequencies very little viscous behaviour is displayed by 
cervical motion segments under axial compression, and the mechanical behaviour is almost entirely elastic.

Conclusions: Motion segments (the disc and the adjacent vertebrae) of the cervical spine display linear behaviour under axial compression, as seen in five cases of isochronal testing. This means that linear viscoelastic models can be applied to the cervical spine. The simplest appropriate linear model showed good agreement with the experimental results when fitted to the data. This suggested most substantial relaxation in the periods around 8 minutes and 5 hours, similar values seen in all of the five cases tested for long term relaxation. A more detailed frequency response is given by Fourier transform mechanical spectroscopy, which showed results consistent with the linear analysis, but did not suggest the presence of any outstanding relaxation times. The loss modulus was seen to decrease smoothly with increasing frequency, while the storage modulus increased. Very little relaxation was seen in the frequency range of $1 \mathrm{~Hz}$ and above; the disc does not function as an effective shock absorber of axial loads at these frequencies. It may be that if the cervical joints are free to flex or extend (as in vivo) then other mechanisms may allow dissipation of energy, for example, extension of ligaments or musculature. This is merely speculation, but may explain why the resonance at $5 \mathrm{~Hz}$ observed in vivo (Panjabi et al., 1986, Paddan and Griffin, 1988) is not seen under these or other axial compression tests (Smeathers, 1989). Clearly the cervical spine dissipates viscous loads over a long time scale, and adapts to changes throughout the course of the day rather than over a few seconds.

\section{References:}

Arridge, R. G. C., and Barham, P. J. (1986). Fourier transform mechanical spectroscopy. Journal of Physics D: Applied Physics, 19, L89-L96.

Aspden, R. M. (1991). Aliasing effects in Fourier transforms of monotonically decaying functions and the calculation of viscoelastic moduli by combining transforms over different time periods. Journal of Physics D: Applied Physics, 24, 803808.

Dorrington, K. L. (1980). The theory of viscoelasticity in biomaterials. Symposia of the Society for Experimental Biology, 34, 289-314.

Holmes, A. D. (1991). Ph.D. Thesis, University of Manchester, Manchester, U.K.

King-Liu, Y., and Guo Dai, Q. (1989). The second stiffest axis of a beam column: implications for cervical spine trauma. Journal of Biomechanical Engineering, 111, 122127.
Paddan, G. S., and Griffin, M. J. (1988). The transmission of translational seat vibration to the head. I: Vertical seat vibration. Journal of Biomechanics, 21, 191-197.

Panjabi, M. M., Andersson, G. B. J., Jorneus, L., Hult, E., and Mattson, L. (1986). In vivo measurements of spinal column vibrations. Journal of Bone and Joint Surgery, 68A, 695-702.

Shames, I. H., and Cozzarelli, F. A. (1992). Elastic and Inelastic Stress Analysis. Prentice Hall, Englewood Cliffs, New Jersey.

Smeathers, J. E., and Joanes, D. N. (1988). Dynamic compressive properties of human lumbar intervertebral joints: a comparison between fresh and thawed specimens. Journal of Biomechanics, 21, 425-433.

Smeathers, J. E. (1989). Measurement for transmissibility for the human spine during walking and running. Clinical Biomechanics, 4, 34-40. 